\title{
Single-approach vertebral osteosynthesis in the treatment of spinal osteolysis by spondylodiscitis
}

\author{
Oriela Rustemi, MD, Fabio Raneri, MD, Lorenzo Alvaro, MD, Luca Gazzola, MD, \\ Giacomo Beggio, MD, Ludovico Rossetto, MD, and Patrizio Cervellini, MD \\ Department of Neurosurgery, San Bortolo Hospital, Vicenza, Italy
}

\begin{abstract}
OBJECTIVE Both spontaneous and iatrogenic spondylodiscitis are becoming ever more frequent, yet there are no definite treatment guidelines. For many years the treatment protocol was conservative medical management or surgical debridement with patients immobilized or bedridden for weeks and often resulting in spinal deformity. The eventual development of spinal deformity can be difficult to treat. Over the last few years, the authors have preferred a single-approach instrumented arthrodesis when spondylolysis that evolves in deformity from somatic wedging occurs.
\end{abstract}

METHODS The authors retrospectively reviewed the clinical, radiological, and surgical records of 11 patients treated over the past 3 years for spondylodiscitis with osteosynthesis.

RESULTS Overall, the authors treated 11 patients: 3 cases with tuberculous spondylodiscitis ( 1 dorsal, 2 lumbar); 6 cases with Staphylococcus aureus spondylodiscitis (1 cervical, 2 dorsal, 2 lumbar, 1 dorsolumbar); 1 spondylodiscitis with postsurgical lumbar deformity; and in 1 dorsolumbar case the germ was not identified. Surgical approaches were chosen according to spinal level: In 8 dorsolumbar cases a posterior osteosynthesis was achieved. In 1 cervical case an anterior approach was performed with autologous bone graft from iliac crest. In 2 thoracolumbar cases a posterolateral costotransversectomy was needed. In 1 lumbosacral case iliac somatic grafting was used. Ten patients received adequate antibiotic treatment with clinical remission, and 1 case is in initial follow-up. No complications due to instrumentation were recorded. Spinal deformity was prevented in 10 cases, whereas preexisting spinal deformity was partially corrected in 1 case. In all cases, arthrodesis achieved vertebral stability.

CONCLUSIONS This study has the limitations of a retrospective review with a limited number of patients. Instrumentation does not appear to hamper healing from infection. Moreover, spinal stabilization, which is assisted by the infectious process even in the absence of bone graft, allows early mobilization. Instrumented osteosynthesis should be preferred for spondylodiscitis with osteolysis and spinal instability because it allows early mobilization and rehabilitation whenever necessary. It prevents spinal deformity and does not hamper healing of infections.

https://thejns.org/doi/abs/10.3171/2018.10.FOCUS18442

KEYWORDS spondylodiscitis; spinal infection; spinal osteolysis; vertebral osteosynthesis

$\mathrm{S}$ PONDYLODISCITIS cases have a low incidence, but they have become more frequent in the last decades..$^{17}$ The incidence of infectious spondylodiscitis in developed countries varies from 4 to 24 cases per million per year. ${ }^{4}$ The aging of the population, immunosuppression, increasing access to diagnostic MRI, and the increasing number of spine surgeries have contributed to the increase in incidence. ${ }^{19}$ Clinical presentation is rather unspecific. The most frequent symptom is insidious and constant back or neck pain in approximately $85 \%$ of the cases, whereas fever may be present in less than half of the patients. ${ }^{4}$ The delay in diagnosis is $2-6$ months from onset of symptoms.
Spondylodiscitis remains a severe disease with a mortality rate ranging from $2 \%$ to $20 \% .^{16}$ The historical treatment options were conservative or local surgical debridement. Instrumented spinal surgery was thought to favor the infectious process. The patients required long periods of immobilization-generally longer than 6 weeks. Spinal deformity outcomes were difficult to treat.

The treatment options are still controversial..$^{10,17}$ There are no current generally applicable treatment guidelines due to the heterogeneity of patients, frequent comorbidities, and large variety of treatment modalities. ${ }^{7}$ One randomized controlled trial proved a strong level of recom- 
mendation for 6 weeks of systemic antibiotic therapy. ${ }^{1}$ Surgical indications are for patients with neurological deficits, spinal deformity, spinal instability, or failure of conservative treatment. Conservative treatment is associated with spinal pain and a higher rate of long-term spinal deformity. ${ }^{10}$ Surgical treatment is associated with perioperative morbidity but with lower long-term mortality. Bydon et al. showed that instrumentation does not increase the rate of recurrent infection if compared to decompression alone. ${ }^{2}$ An increasing number of studies have shown that instrumented spine surgery for spinal infections is a safe procedure..$^{3,3,5-9,12-15}$ In recent years, we have considered surgical arthrodesis with a single approach for spondylodiscitis with lysis of the vertebral body as evidence of vertebral deformity.

\section{Methods}

This was a retrospective study of patients treated between June 2015 and June 2018. We reviewed clinical records, radiological data, and surgical notes obtained for all patients undergoing surgical osteosynthesis for spondylodiscitis with lysis of the vertebral body and tendency to deformity at our institution. Eleven patients fulfilled the inclusion criteria. We reviewed patient demographic data such as age and sex, presenting symptoms and neurological examination, radiographic images, operative reports, postoperative complications, and clinical and radiological follow-up data. The choice of the approach depended on the level of spondylodiscitis. We preferred a single-step approach in all cases. For cervical spondylodiscitis we chose an anterior approach, whereas a posterior approach for lumbar cases or a posterolateral approach for some of the dorsolumbar cases was preferred. We did not find more complex multiple approaches necessary for these patients on preoperative study. Titanium instrumentation was used for dorsal and lumbar cases, and iliac crest graft was used for the cervical case and for one of the lumbar cases. Results of CT-guided needle biopsy were recorded when performed. Referring infectious disease specialists decided on antibiotic therapy. Initial therapy required broad-spectrum intravenous antibiotics. Once complete microbiology data were available, if a germ could be identified a specific antibiotic therapy was followed. The switch to oral antibiotics depended on adequate clinical and blood response.

\section{Results}

Eleven patients with spondylodiscitis and lysis of the vertebral body were treated with osteosynthesis. The mean age was 62 years (range 29-76 years), and there were 8 female and 3 male patients. The etiological agents were $M y$ cobacterium tuberculosis in 3 cases (1 dorsal, 2 lumbar); Staphylococcus aureus in 6 cases (1 cervical, 2 dorsal, 2 lumbar, 1 dorsolumbar); S. epidermidis and S. capitis spondylodiscitis in 1 case as a complication in a postsurgical lumbar deformity; and the germ was not identified in 1 dorsolumbar case. Surgical approaches were chosen according to spinal level: in 8 thoracic or lumbar cases a posterior approach was preferred; in 1 cervical case an anterior approach with iliac crest grafting was performed; in
2 cases at the dorsolumbar level a posterolateral approach (costotransversectomy) was chosen; and in 1 lumbosacral case iliac somatic grafting was used.

The anterior spinal column was reconstructed with iliac crest bone grafting in only 2 cases: in the patient with cervical spondylodiscitis (case 5) and in the patient with postsurgical infection in the L5-S1 level (case 10) (Table 1). The remainder of the thoracolumbar cases underwent a posterior or posterolateral arthrodesis. The general clinical conditions rendered these patients too weak for longer or combined approaches. The anterior spinal column was not reconstructed and we initially planned to reconstruct it in a second-step surgery after stabilization of the general conditions. However, at follow-up the patients showed good clinical recovery. In some cases, calcific bone bridges were assessed as fusion on CT scans and in the remainder the deformity was prevented. We decided not to proceed with a second surgery for reconstruction of the anterior columns in any of the cases. An orthopedic brace was used for 8-12 weeks in all cases. Despite the absence of anterior column direct reconstruction in thoracolumbar cases, spinal deformity was prevented at last follow-up in 10 cases, and preexisting spinal deformity was partially corrected in 1 case.

Three patients presented with neurological deficits and improved after surgery. The remaining 8 patients presented with spinal pain that was more responsive to analgesic treatment after vertebral stabilization, and they showed no pain at follow-up. The follow-up range was 3-36 months (average 16 months). None of the patients had new-onset neurological deficits after surgery.

All patients without neurological deficits started mobilization on day 2 after surgery, after the paravertebral drainage devices were removed, and after postoperative CT scans showed the correct screw positioning. An orthopedic brace was used for 8-12 weeks. Patients with neurological deficits started early rehabilitation. Table 1 summarizes the clinical characteristics of the patients.

Two patients (cases 7 and 8) had superficial infection of the surgical wound, which resolved after surgical revision. Case 7 presented with surgical wound dehiscence and infection from Staphylococcus. A surgical revision was performed at 20 days after the stabilization surgery. Purulent material was present in the muscle layers and it was removed with multiple washings with physiological solutions containing betadine and $\mathrm{H}_{2} \mathrm{O}_{2}$. We used vacuumassisted closure, which helped with wound healing.

Case 8 presented with external signs of wound infection. Surgical revision at 20 days after surgery showed superficial infection from Pseudomonas. We used multiple washings with physiological solutions containing betadine and $\mathrm{H}_{2} \mathrm{O}_{2}$. The surgical wound healed without any additional intervention.

The patients were quickly transferred to an infectious disease unit or a rehabilitation unit according to clinical conditions. Nutritional support was evaluated on a casespecific basis. All patients received 6 weeks of systemic antibiotic therapy decided by the referring infectious disease specialists. Risk factors in this cohort included elderly debilitated patients with systemic blood infection, decompensated diabetes mellitus, pulmonary tuberculosis, 
TABLE 1. Clinical characteristics of 11 patients with spinal osteolysis

\begin{tabular}{|c|c|c|c|c|c|c|}
\hline $\begin{array}{l}\text { Case } \\
\text { No. }\end{array}$ & $\begin{array}{l}\text { Age } \\
\text { (yrs), } \\
\text { Sex }\end{array}$ & $\begin{array}{l}\text { Spondylodiscitis } \\
\text { Level }\end{array}$ & $\begin{array}{l}\text { Preop Neurological } \\
\text { Deficits }\end{array}$ & Infective Agent & Surgical Procedure & Neurological Exam at Follow-Up \\
\hline 1 & $76, \mathrm{M}$ & L1-2 & $\begin{array}{l}\text { Deficit in rt foot dorsi- } \\
\text { flexion; lumbar pain }\end{array}$ & M. tuberculosis & $\begin{array}{l}\text { Microdiscectomy, debride- } \\
\text { ment, arthrodesis }\end{array}$ & $\begin{array}{l}\text { Good recovery of strength in lower } \\
\text { limbs; no pain }\end{array}$ \\
\hline 2 & $67, \mathrm{~F}$ & T10-11 & None & S. aureus & $\begin{array}{l}\text { T8-T9-T12-L1 osteosynthesis; } \\
\text { T9-10 laminectomy }\end{array}$ & No deficits \\
\hline 3 & $74, \mathrm{~F}$ & T8-9 & Severe paraparesis & S. aureus & $\begin{array}{l}\text { Lt laminarthrectomy T8-9, } \\
\text { decompression, T6-11 } \\
\text { arthrodesis }\end{array}$ & Improvement in lower-limb mobility \\
\hline 4 & $68, M$ & L4-5 & None & S. aureus & $\begin{array}{l}\text { L3-L4-L5 arthrodesis; laminec- } \\
\text { tomy of L4, L5, \& partial L3 }\end{array}$ & No deficits \\
\hline 5 & $68, F$ & C4-5 & None & S. aureus & $\begin{array}{l}\text { C3 \& C4 somatectomy, iliac } \\
\text { crest graft }\end{array}$ & No deficits, no pain \\
\hline 6 & $64, \mathrm{~F}$ & $\begin{array}{l}\text { T12-L2 } \\
\text { deformity } \\
\text { (kyphosis) }\end{array}$ & None & S. aureus & $\begin{array}{l}\text { T9-L4 arthrodesis, laminecto- } \\
\text { my, bilat T12, L1 arthrec- } \\
\text { tomy, partial L2 arthrectomy }\end{array}$ & $\begin{array}{l}\text { Partial correction of kyphosis, no } \\
\text { deficits }\end{array}$ \\
\hline 7 & $61, F$ & T9-11 & $\begin{array}{l}\text { Severe paraparesis, } \\
\text { almost paraple- } \\
\text { gia; uncontrolled } \\
\text { sphincters }\end{array}$ & M. tuberculosis & $\begin{array}{l}\text { T8-T9-T12-L1 arthrodesis; } \\
\text { T10-11 laminectomy }\end{array}$ & $\begin{array}{l}\text { Surgical wound infection w/ Staphy- } \\
\text { lococcus; debridement at } 20 \text { days } \\
\text { from stabilization; improvement } \\
\text { at } 5 \text { mos, walking w/ support }\end{array}$ \\
\hline 8 & $74, \mathrm{~F}$ & T10-11, L3 & None & $\begin{array}{l}\text { Infectious process, } \\
\text { no germ identified }\end{array}$ & T8-L4 posterior arthrodesis & $\begin{array}{l}\text { Surgical wound infection w/ Pseu- } \\
\text { domonas; debridement }\end{array}$ \\
\hline 9 & $56, F$ & L2-3 & None & M. tuberculosis & $\begin{array}{l}\text { L1-L2-L3-L4 arthrodesis, rt } \\
\text { L2-3 decompression \& } \\
\text { microdiscectomy }\end{array}$ & No deficits, no pain \\
\hline 10 & $44, \mathrm{M}$ & $\begin{array}{l}\text { L5-S1, postsur- } \\
\quad \text { gical }\end{array}$ & None & $\begin{array}{l}\text { S. capitis, S. epider- } \\
\text { midis }\end{array}$ & $\begin{array}{l}\text { Posterolateral L4-S1 arthrod- } \\
\text { esis, iliac crest grafting, } \\
\text { L5-S1 disc debridement, } \\
\text { from It-sided approach }\end{array}$ & No deficits, no pain \\
\hline 11 & $29, \mathrm{~F}$ & L3 & None & S. aureus & $\begin{array}{l}\text { L2-4 arthrodesis, rt L2 hemi- } \\
\text { laminectomy }\end{array}$ & No deficits \\
\hline
\end{tabular}

eating disorders in a young patient, previous spine surgery in 1 case, and hepatitis.

In 10 patients antibiotic treatment led to the resolution of the infectious process despite the presence of synthetic material, and 1 case is still in initial follow-up. In 1 case a deformity already present was partially corrected, and in the remaining 10 cases the vertebral deformity was prevented. In all cases, the arthrodesis permitted vertebral stability.

Vertebral fusion was ascertained with the observation of calcific bone bridges on follow-up spinal CT in outpatient evaluation. Radiological fusion was assessed in the patients with iliac bone grafting. Despite the fact that not all of the thoracolumbar cases showed radiological fusion, none of them showed screw or rod breaks or dislocation. The short length of follow-up is the main limitation of this study.

\section{Illustrative Cases \\ Case 5}

A 68-year-old woman presented with bilateral cervicobrachialgia. An MRI study obtained with contrast showed enhancement in the third and fourth cervical body (C3 and $\mathrm{C} 4$ ) and the interposed disc, with epidural abscess and cervical spinal cord compression (Fig. 1 left). An anterior approach was performed with $\mathrm{C} 3$ and $\mathrm{C} 4$ somatectomy and iliac crest grafting. The pathogenic agent was $S$. aureus. The infectious process resolved after antibiotic therapy. The patient did not present with pain, and the results of her neurological examination were normal. The control CT scan showed vertebral stability (Fig. 1 right).

\section{Case 6}

A 64-year-old woman presented with dorsolumbar pain. Admission T2-weighted MRI showed altered signal in L1 with lysis of the vertebral body as a sign of instability; altered signal was also present in the discs between L5S1 and T10-11, consistent with spondylodiscitis (Fig. 2A). The patient refused stabilization surgery. She returned after 8 months with failure of conservative treatment: MRI showed complete collapse of the soma of L5, altered signal in T12-L2, and severe kyphosis (Fig. 2B). T9-L4 arthrodesis, laminectomy, bilateral T12, L1 arthrectomy, and partial L2 arthrectomy were performed. The patho- 

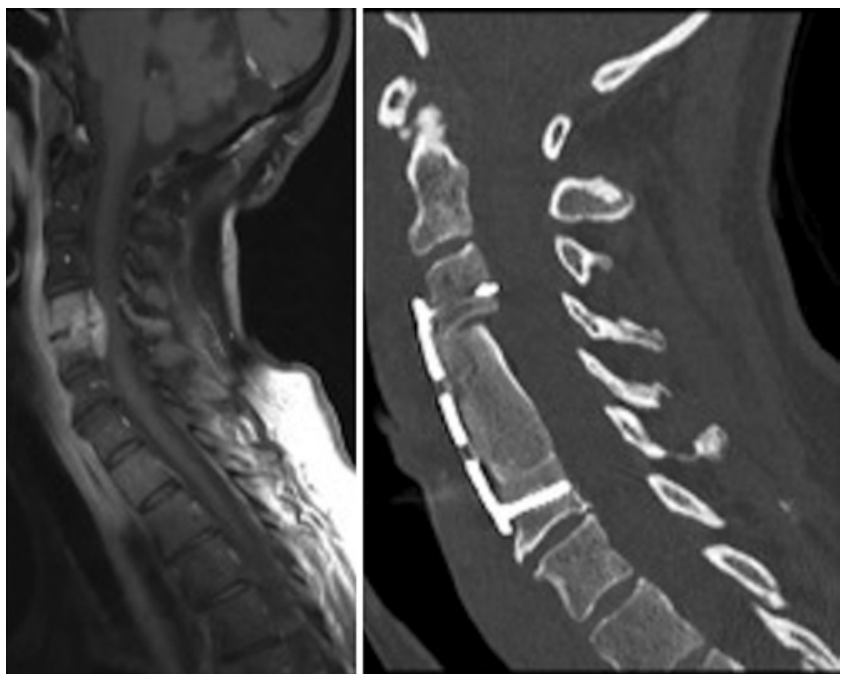

FIG. 1. Case 5. Left: MRI showing contrast enhancement in C3, C4, and the interposed disc, with epidural abscess and cervical spinal cord compression. Right: Postoperative CT scan obtained after C3 and C4 somatectomy and iliac crest grafting. The pathogenic agent was $S$. aureus.

genic agent was $S$. aureus. The infectious process resolved after antibiotic therapy. Results of the neurological examination were normal. The control CT scan showed partial correction of the vertebral deformity (Fig. 2C).
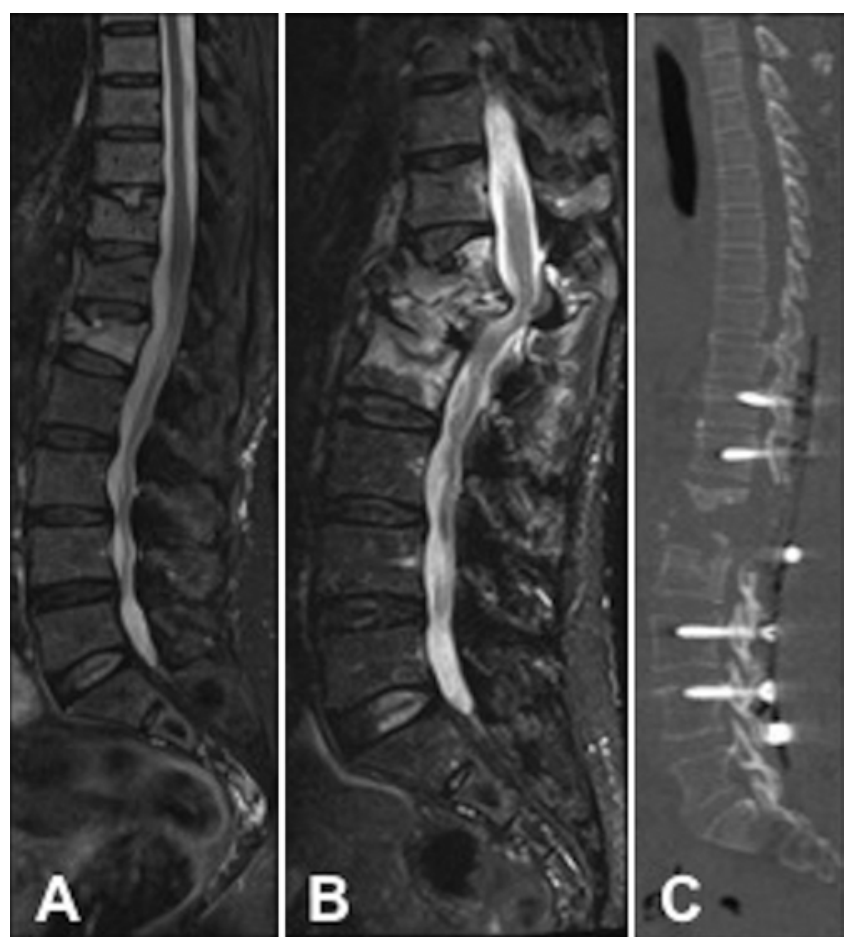

FIG. 2. Case 6. A: T2-weighted MRI showing altered signal in L1 with lysis of the vertebral body as instability sign, and altered signal was also present in the discs between L5-S1 and T10-11, consistent with spondylodiscitis. The patient refused stabilization surgery. B: MRI study obtained after 8 months showing complete collapse of the soma of $L 5$, altered signal in T12-L2, and severe kyphosis. C: Postoperative CT showing partial correction of the vertebral deformity. The pathogenic agent was $S$. aureus.
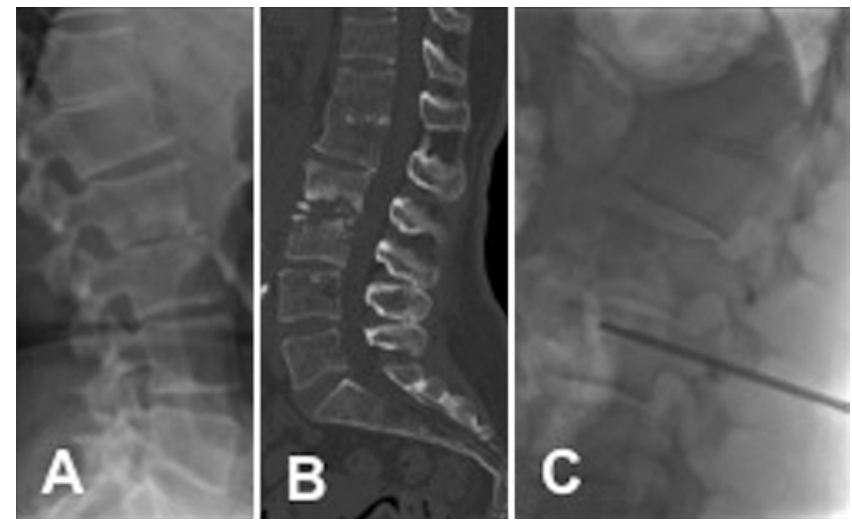

FIG. 3. Case 9. A: Radiographic image showing deformity. B: CT scan showing reduction of the intersomatic space in L2-3 and lysis of the somatic interposed plate as signs of instability. C: The needle biopsy sample was negative for pathogens. The pathogen was identified after a new, more consistent sample was obtained with surgery.

\section{Case 9}

A 56-year-old woman had experienced lumbar pain in the last 6 months and fever at the onset of the pain. Radiographs (Fig. 3A) and CT scans (Fig. 3B) showed reduction of the intersomatic space at L2-3 and lysis of the somatic interposed plate as a sign of instability. Results of the needle biopsy were negative (Fig. 3C). MRI showed contrast enhancement in the vertebral body of L2 and L3 as a sign of infection (Fig. 4 left). L1-L2-L3-L4 arthrodesis, right L2-3 decompression, and microdiscectomy were performed. The infective agent was $M$. tuberculosis. The infectious process resolved after antibiotic therapy. The patient did not present with pain and results of the neurological examination were normal. The control CT scan showed vertebral stability (Fig. 4 right).

\section{Case 10}

A 44-year-old man who had undergone previous microdiscectomy for a herniated disc showed vertebral infection resistant to medical therapy alone with progressive vertebral lysis (T2 MRI; Fig. 5A and B). The patient underwent posterolateral L4-S1 arthrodesis, iliac crest grafting, and L5-S1 disc debridement from a left-sided approach. The infective agents were S. epidermidis and $S$. capitis. The infectious process resolved after antibiotic therapy. The patient did not present with pain and results of the neurological examination were normal. The control CT scan showed vertebral stability (Fig. 5C).

\section{Discussion}

Although generally rare, cases of spondylodiscitis have increased in frequency in Western countries over the last decades. They have become an issue that neurosurgeons have to face more frequently. In the past, treatment was generally conservative with long periods of immobilization, and delayed deformities were quite difficult to treat. Instrumentation in patients with spinal infection was a taboo, because of the perceived risk of promoting bacterial superinfection of the foreign bodies. Recently, many 

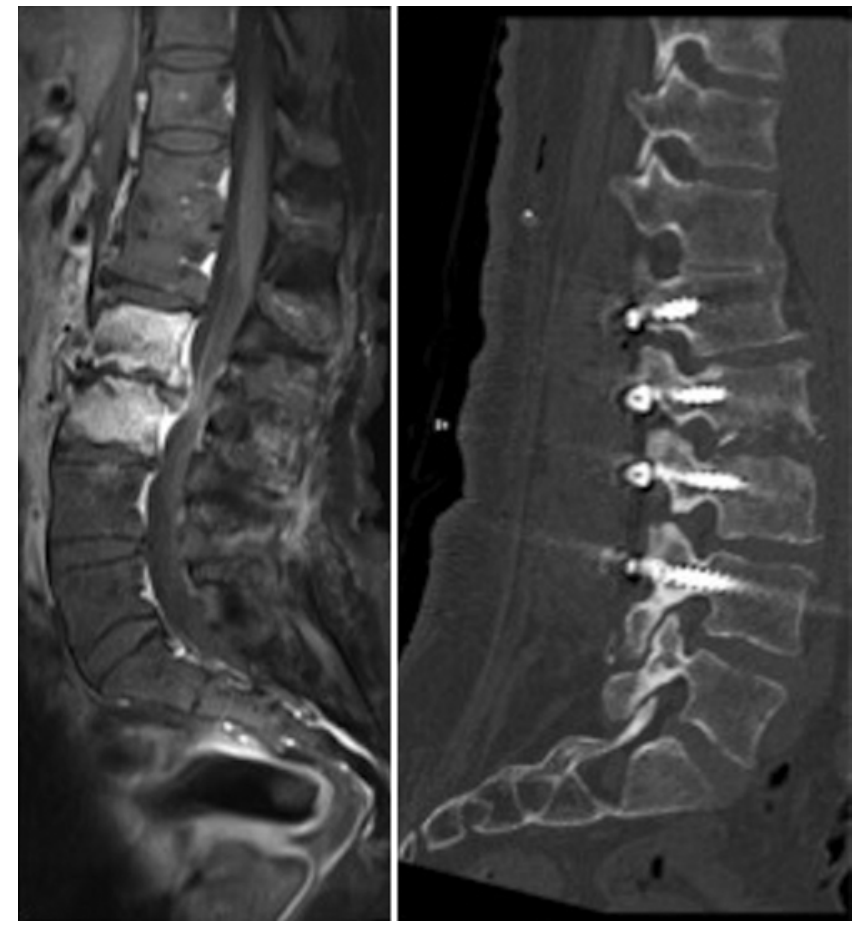

FIG. 4. Case 9. Left: MRI study showing contrast enhancement in the vertebral body of $L 2$ and $L 3$ as a sign of infection. Right: Postoperative CT scan. The infective agent was $M$. tuberculosis.

independent studies have shown that instrumented spine surgery for spinal infections is a safe and feasible option. , $3,5-9,12-15$ Conversely, spinal stabilization could even promote accelerated healing. ${ }^{9}$ The risk of reoperation for infection in patients with instrumentation for spinal infection is $\leq 10 \%$. $^{18}$ Than and Mummaneni ${ }^{18}$ suggested that instrumentation in the setting of primary infection should be considered when there is a clinical indication.

Figure 6 illustrates the general treatment algorithm we apply for spondylodiscitis. Three patients in the present series presented with neurological deficits; thus, acute surgical decompression was mandatory. These patients had vertebral instability for which they needed instrumented stabilization. The screws were positioned prior to decompression in the same surgical approach to maintain the surgical anatomical references. These patients improved neurologically after lengthy rehabilitation, including a patient who presented as almost paraplegic.

We excluded from surgery patients presenting with severe sepsis who were unable to undergo the surgical procedure. Age was not a standalone discriminant factor for surgical exclusion, although some elderly patients were excluded from surgery after anesthesiology evaluation, despite an infectious process.

Surgical stabilization was performed immediately after referral for the following reasons: first, we wanted to operate when the patient could withstand general anesthesia and before instauration of sepsis. The second reason was to avoid delayed vertebral deformities. Indeed, one patient who refused surgery at presentation returned 8 months later with evidence of spondylodiscitis and a severe deformity that could be corrected only partially.
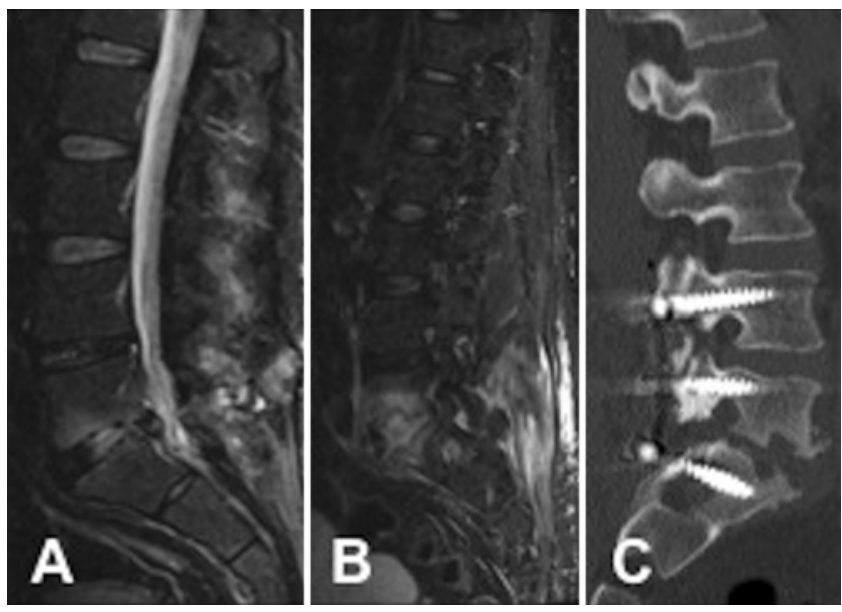

FIG. 5. Case 10. A: MRI showing vertebral infection in a patient who had undergone previous microdiscectomy. B: MRI sequence showing progressive vertebral lysis. C: Postoperative CT control scan. The infective agents were $S$. epidermidis and $S$. capitis.

A single approach was used in all cases. Excluding the case with long-term deformity, a single approach was sufficient to guarantee vertebral stability and avoid deformity. More complex, 2-stage, anterior plus posterior approaches were avoided due to the fragile condition of patients. Longer follow-up and larger series are necessary to better evaluate this strategy.

Staphylococcus aureus (55\% of the cases) was the most common infective agent, concordant with epidemiological data, ${ }^{11}$ followed by M. tuberculosis (27\% of cases). In 1 patient $(9 \%)$ the infective agent could not be identified, and this is reported in up to one-third of the cases in the literature.

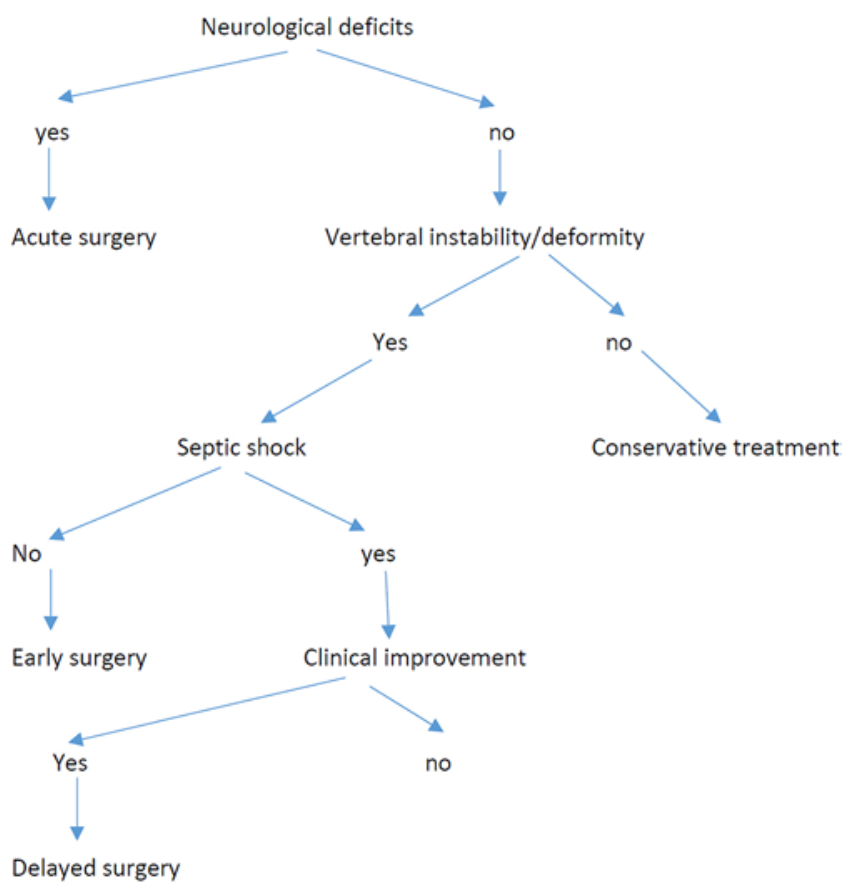

FIG. 6. General treatment algorithm for spondylodiscitis. 
Two patients had a wound infection, which proved to be superficial after revision. No patient had superinfection of the implanted system, and this is consistent with the previously reported studies.

\section{Conclusions}

This study has the limitations of a retrospective review and a limited number of patients. Nevertheless, we found that the presence of synthetic material does not seem to hinder the healing of the infectious process. The stabilization allows an early mobilization of the patient. The infection facilitates arthrodesis even in the absence of bone grafts. A single approach is sufficient to maintain vertebral stability. In the presence of spondylodiscitis, if there is an osteolysis and instability it is advisable to perform osteosynthesis, which allows rapid mobilization, prevents deformity, and does not significantly impede the efficacy of antibiotics.

\section{References}

1. Bernard L, Dinh A, Ghout I, Simo D, Zeller V, Issartel B, et al: Antibiotic treatment for 6 weeks versus 12 weeks in patients with pyogenic vertebral osteomyelitis: an openlabel, non-inferiority, randomised, controlled trial. Lancet 385:875-882, 2015

2. Bydon M, De la Garza-Ramos R, Macki M, Naumann M, Sciubba DM, Wolinsky JP, et al: Spinal instrumentation in patients with primary spinal infections does not lead to greater recurrent infection rates: an analysis of 118 cases. World Neurosurg 82:e807-e814, 2014

3. Carragee E, Iezza A: Does Acute placement of instrumentation in the treatment of vertebral osteomyelitis predispose to recurrent infection: long-term follow-up in immune-suppressed patients. Spine (Phila Pa 1976) 33:2089-2093, 2008

4. Gouliouris T, Aliyu SH, Brown NM: Spondylodiscitis: update on diagnosis and management. J Antimicrob Chemother 65 (Suppl 3):iii11-iii24, 2010

5. Hassan K, Elmorshidy E: Anterior versus posterior approach in surgical treatment of tuberculous spondylodiscitis of thoracic and lumbar spine. Eur Spine J 25:1056-1063, 2016

6. Lee MC, Wang MY, Fessler RG, Liauw J, Kim DH: Instrumentation in patients with spinal infection. Neurosurg Focus 17(6):E7, 2004

7. Lener S, Hartmann S, Barbagallo GMV, Certo F, Thomé C, Tschugg A: Management of spinal infection: a review of the literature. Acta Neurochir (Wien) 160:487-496, 2018

8. Ogden AT, Kaiser MG: Single-stage debridement and instrumentation for pyogenic spinal infections. Neurosurg Focus 17(6):E5, 2004

9. Rayes M, Colen CB, Bahgat DA, Higashida T, Guthikonda M, Rengachary S, et al: Safety of instrumentation in patients with spinal infection. J Neurosurg Spine 12:647-659, 2010

10. Rutges JP, Kempen DH, van Dijk M, Oner FC: Outcome of conservative and surgical treatment of pyogenic spondylodiscitis: a systematic literature review. Eur Spine J 25:983-999, 2016

11. Sapico FL: Microbiology and antimicrobial therapy of spinal infections. Orthop Clin North Am 27:9-13, 1996
12. Schomacher M, Finger T, Koeppen D, Süss O, Vajkoczy P, Kroppenstedt S, et al: Application of titanium and polyetheretherketone cages in the treatment of pyogenic spondylodiscitis. Clin Neurol Neurosurg 127:65-70, 2014

13. Schuster JM, Avellino AM, Mann FA, Girouard AA, Grady MS, Newell DW, et al: Use of structural allografts in spinal osteomyelitis: a review of 47 cases. J Neurosurg 93 (1 Suppl):8-14, 2000

14. Shetty AP, Aiyer SN, Kanna RM, Maheswaran A, Rajasekaran S: Pyogenic lumbar spondylodiscitis treated with transforaminal lumbar interbody fusion: safety and outcomes. Int Orthop 40:1163-1170, 2016

15. Shiban E, Janssen I, da Cunha PR, Rainer J, Stoffel M, Lehmberg J, et al: Safety and efficacy of polyetheretherketone (PEEK) cages in combination with posterior pedicel screw fixation in pyogenic spinal infection. Acta Neurochir (Wien) 158:1851-1857, 2016

16. Skaf GS, Domloj NT, Fehlings MG, Bouclaous CH, Sabbagh AS, Kanafani ZA, et al: Pyogenic spondylodiscitis: an overview. J Infect Public Health 3:5-16, 2010

17. Stüer C, Stoffel M, Hecker J, Ringel F, Meyer B: A staged treatment algorithm for spinal infections. J Neurol Surg A Cent Eur Neurosurg 74:87-95, 2013

18. Than KD, Mummaneni PV: Spinal instrumentation in the setting of primary infection. World Neurosurg 82:e713e714, 2014

19. Valancius K, Hansen ES, Høy K, Helmig P, Niedermann $\mathrm{B}$, Bünger C: Failure modes in conservative and surgical management of infectious spondylodiscitis. Eur Spine J 22:1837-1844, 2013

\section{Disclosures}

The authors report no conflict of interest concerning the materials or methods used in this study or the findings specified in this paper.

\section{Author Contributions}

Conception and design: Rustemi, Rossetto, Cervellini. Acquisition of data: Rustemi, Raneri. Analysis and interpretation of data: all authors. Drafting the article: Rustemi, Raneri. Critically revising the article: all authors. Reviewed submitted version of manuscript: all authors. Approved the final version of the manuscript on behalf of all authors: Rustemi. Administrative/technical/material support: Rustemi, Cervellini. Study supervision: Rustemi, Cervellini.

\section{Supplemental Information Previous Presentations}

An abstract was published in the European Spine Journal, Volume 27, Number 4, April 2018; and in the section Abstracts of the SICV (Italian Society of Vertebral Surgery) and GIS (Italian Scoliosis Group) Meeting, Cagliari, Italy, May 10-12, 2018.

\section{Correspondence}

Oriela Rustemi: San Bortolo Hospital, Vicenza, Italy. orielarustemi@libero.it. 